Mechanical Orientation in Thermotropic Liquid Crystal State and Magnetic Orientation in Solvent Evaporation Process via Lyotropic Liquid Crystal State of an Amphotropic Low-Bandgap Liquid Crystalline $\pi$-Conjugated Polymer

Hiromasa Goto, ${ }^{1, *}$ Aohan Wang, ${ }^{1}{ }^{1}$ Shigeki Nimori, ${ }^{2}$ Kohsuke Kawabata

Liquid Crystal, 2013, 40(9), 1159-1166.

\title{
Mechanical Orientation in Thermotropic Liquid Crystal State and Magnetic Orientation in Solvent Evaporation Process via Lyotropic Liquid Crystal State of an Amphotropic Low-Bandgap Liquid Crystalline $\pi$-Conjugated Polymer
}

\author{
Hiromasa Goto, ${ }^{1, *}$ Aohan Wang, ${ }^{1}{ }^{1}$ Shigeki Nimori, ${ }^{2}$ Kohsuke Kawabata ${ }^{1}$
}

\author{
${ }^{1}$ Division of Materials Science, Faculty of Pure and Applied Sciences, University of \\ Tsukuba, Tsukuba, Ibaraki 305-8573, Japan \\ ${ }^{2}$ Tsukuba Magnet Laboratory, National Institute for Materials Science, Tsukuba, \\ Ibaraki, 305-0003, Japan
}

*Correspondence to H. Goto, email: gotoh@ims.tsukuba.ac.jp, fax: +81-298-53-4490

\begin{abstract}
Molecular orientations by shear stress and magnetic field were carried out to obtain a uniaxial, low-bandgap, amphotropic liquid crystalline (LC) $\pi$-conjugated polymer. Orientation of a thick polymer film shows orientation along the shear stress, but a thin sample produced by a spatula drawing on a glass substrate produces LC band-structure with a chevron pattern. Furthermore, we report orientation of a lyotropic polymer LC under magnetic field.
\end{abstract}

Keywords: liquid crystal, band structure, low-bandgap, chevron structure, uniaxial orientation

\section{Introduction}

Liquid crystals (LC) show interesting properties, such as aggregation and orientation. Liquid crystal alignment is one of the most important issues in materials chemistry because aligned polymers show improved mechanical strength and optical anisotropy. ${ }^{1-7}$ Conjugated polymers have been employed in electronic devices, such as electroluminescence displays ${ }^{8-11}$ and solar cells. ${ }^{12-14}$ Liquid crystalline semiconductors were developed to obtain opto-electronic functional materilas. ${ }^{15-17}$ Optoelectronic materials having optical anisotropy may find use in polarized optoelectronic devices. 
Mechanical Orientation in Thermotropic Liquid Crystal State and Magnetic Orientation in Solvent Evaporation Process via Lyotropic Liquid Crystal State of an Amphotropic Low-Bandgap Liquid Crystalline $\pi$-Conjugated Polymer

Hiromasa Goto, ${ }^{1, *}$ Aohan Wang, ${ }^{1}{ }^{1}$ Shigeki Nimori, ${ }^{2}$ Kohsuke Kawabata

Liquid Crystal, 2013, 40(9), 1159-1166.

We have studied molecular alignment of LC conjugated polymers by magnetic field ${ }^{18}$ and rubbing technique. ${ }^{19}$ Recently, alignment of a liquid crystalline conjugated polymer was obtained by combination of capillary technique and solution casting. ${ }^{20}$

In nature, some desert beetles determine direction at night by detecting polarized light from the moon ${ }^{21,22}$; mantis shrimp correctly recognize circularly polarized light signals in the sea. ${ }^{23}$ Beetles selectively reflect circularly polarized light to show metallic color reflections. ${ }^{24}$ Therefore, it is highly promising to mimic nature and adapt opto-functional technology from biological systems.

We have studied orientation of liquid crystalline conjugated polymers. Synthesis of the polymer employed in this study was previously carried out. ${ }^{25}$ The polymer shows both lyotropic and thermotropic liquid crystalline properties (amphotropic). In the present study, mechanical orientation of the polymer with shear stress in the thermotropic liquid crystal (LC) state was carried out. Furthermore, we developed a method of magnetic orientation in solvent evaporation process via lyotropic LC state (lyotropic LC magnetic orientation in evaporation process, LME). In the evaporation process, the polymer shows lyotropic LC at appropriate concentration. The lyotropic LC state has good susceptibility for magnetic field. The polymer is aligned under magnetic field in lyotropic LC state. Completion of evaporation allows formation of uniaxial orientated solid sample with LC order.

This paper focuses on the development of orientation methods for conjugated polymers.

\section{Results and Discussion}

\section{Polymer structure}

The polymer structure employed in this study is shown in Figure 1. The polymer consists of an isothianaphthene unit and a phenylene unit having two mesogens in the side chains (abbreviated as polyITN-LC). The isothianaphthene unit of the copolymer provides low bandgap, and the LC unit imparts liquid crystallinity. This combination provides low bandgap LC properties for polyITN-LC. 
Mechanical Orientation in Thermotropic Liquid Crystal State and Magnetic Orientation in Solvent Evaporation Process via Lyotropic Liquid Crystal State of an Amphotropic Low-Bandgap Liquid Crystalline $\pi$-Conjugated Polymer

Hiromasa Goto, ${ }^{1, *}$ Aohan Wang, ${ }^{1}{ }^{1}$ Shigeki Nimori, ${ }^{2}$ Kohsuke Kawabata

Liquid Crystal, 2013, 40(9), 1159-1166.

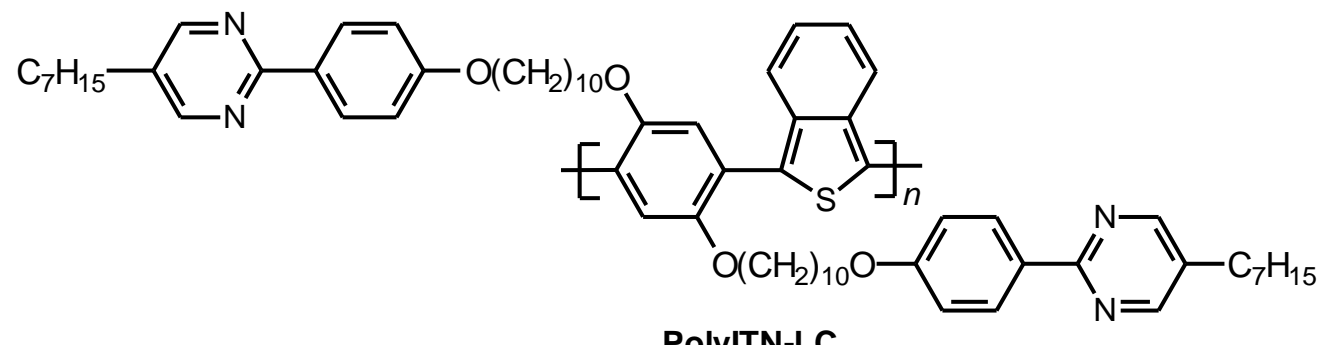

Figure 1.Molecular structure of polyITN-LC.

\section{Thermotropic LC properties}

The polymer shows thermotropic liquid crystallinity. An optical texture obtained with polarizing optical microscopy (POM) for the polyITN-LC at $60{ }^{\circ} \mathrm{C}$ is shown in Figure 2(a). During heating process, the polyITN-LC shows fan-shaped texture with red color due to the $\pi-\pi^{*}$ transition of the main-chain. Retardation color of the polymer under the POM did not appear because the intrinsic color of the polyITN-LC derived from the conjugated main chain mainly appears as dark red. PolyITN-LC shows a mesophase between $12{ }^{\circ} \mathrm{C}$ and $90{ }^{\circ} \mathrm{C}$ during heating, and $12{ }^{\circ} \mathrm{C}$ and $78{ }^{\circ} \mathrm{C}$ during cooling. ${ }^{23}$

\section{Orientation with shear stress}

Liquid crystals can be aligned by shear stress, magnetic field, or electric field. Figure 2(b) shows mechanically oriented polyITN-LC at LC temperature (ca. $60{ }^{\circ} \mathrm{C}$ ). The sample was sandwiched between two glass plates, and heated to the LC temperature range [Figure. 2(c)]. Then, the two glass plates were moved in opposite directions to apply shear stress to the sandwiched sample. The LC sample thus prepared shows uniaxial alignment, which is observable under the POM. The SmA LC multi-domains are aligned in one direction parallel to the plates' motion. It should be noted that mono-domain samples generally show no characteristic structure because the entire region forms one domain. Although no mono-domain (single domain) structure of the present polymer was obtained by the shear stress, uniaxial alignment of the multi-domains was achieved. 
Mechanical Orientation in Thermotropic Liquid Crystal State and Magnetic Orientation in Solvent Evaporation Process via Lyotropic Liquid Crystal State of an Amphotropic Low-Bandgap Liquid Crystalline $\pi$-Conjugated Polymer

Hiromasa Goto, ${ }^{1, *}$ Aohan Wang, ${ }^{1}{ }^{1}$ Shigeki Nimori, ${ }^{2}$ Kohsuke Kawabata Liquid Crystal, 2013, 40(9), 1159-1166.

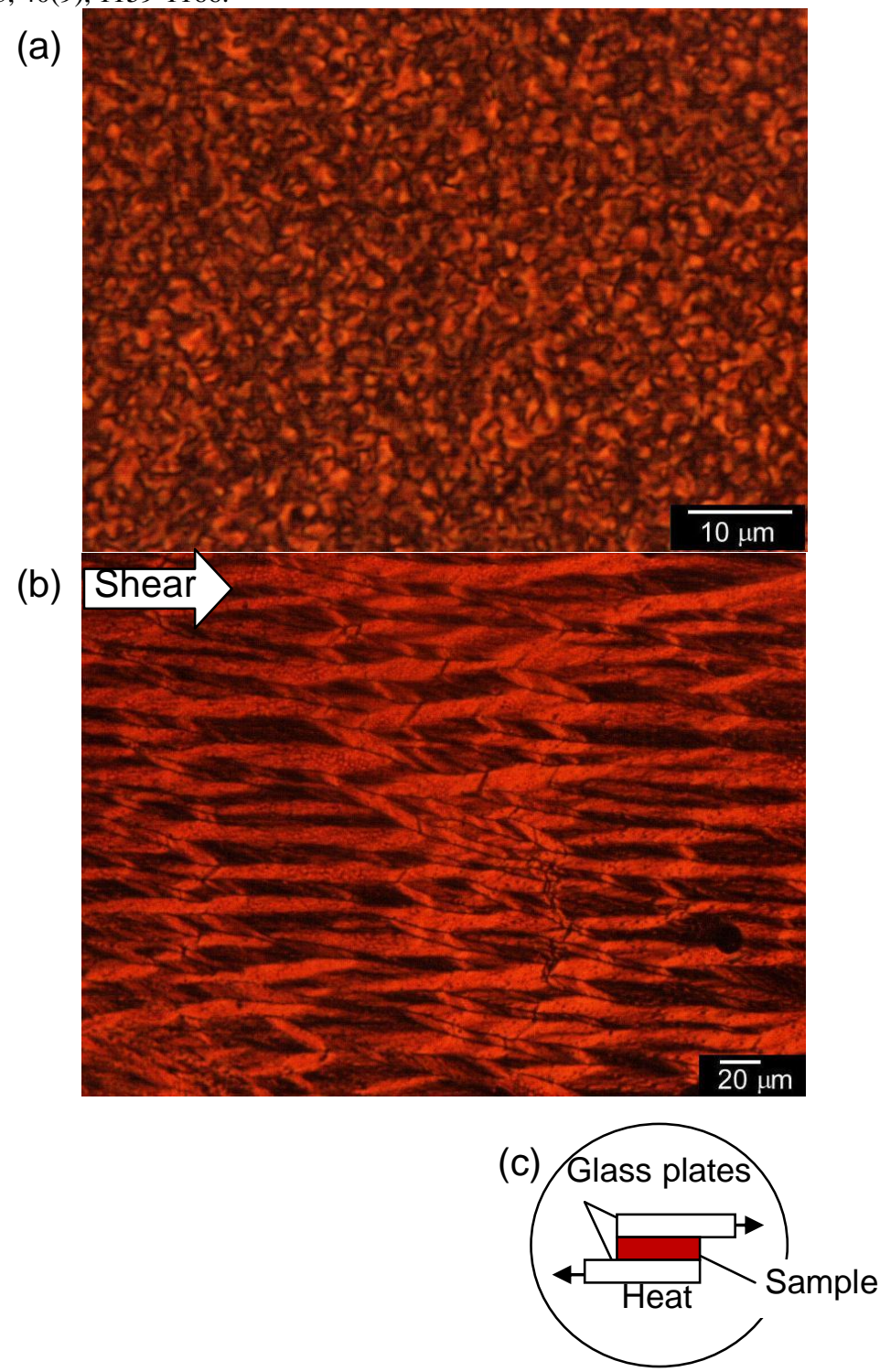

Figure 2. Polarising optical microscopy (POM) image of the oriented polyITN-LC (thick sample) obtained under shear stress. The orientation was accomplished by mechanically sliding glass plates. (a) POM image of the sample after orientation in thermotropic liquid crystalline state, (b) mechanical orientation between two glass plates to obtain oriented sample and (c) sliding plate geometry.

Furthermore, we carried out mechanical orientation of polyITN-LC with a spatula, as shown in Figure 3(a). The shear stress affords thin, oriented samples of polyITN-LC showing uniaxial alignment. The SmA LC domains sequentially arrange and the entire arrangement shows line formation, which is observable in Figure 3(b). Magnification of this image indicated that the domains are oriented in the vertical direction against the 
Mechanical Orientation in Thermotropic Liquid Crystal State and Magnetic Orientation in Solvent Evaporation Process via Lyotropic Liquid Crystal State of an Amphotropic Low-Bandgap Liquid Crystalline $\pi$-Conjugated Polymer

Hiromasa Goto, ${ }^{1, *}$ Aohan Wang, ${ }^{1}{ }^{1}$ Shigeki Nimori, ${ }^{2}$ Kohsuke Kawabata

Liquid Crystal, 2013, 40(9), 1159-1166.

shear direction (Figure 3(c)). This structure is called "band-structure of LCs". In this

case, chevron band structure was obtained for polyITN-LC (Figure 3(d)). Figure S1

(Supplementary Information) displays oriented polyITN-LC with insertion of gypsum first-order red plate under cross Nicol condition. The colorful, oriented domain structure is derived from retardation. As for the thick sample, optical retardation color under cross Nicol condition was covered by the intrinsic red color of the polymer. Therefore, the thick sample shows mono-color of red. On the other hand, the thin sample mainly displays multi-colour optical retardation. It should be noted that the shear between glass plates is a simple shear, while deformation with a spatula is a combination of simple shear and elongational flow.

\section{Lyotropic LC properties}

This polymer shows lyotropic liquid crystallinity in tetrahydrofuran (THF), chloroform, dichloromethane, and toluene solutions. Evaporation of solvents from the polymer solution yields solid polymer films with LC domains via lyotropic LC phase. We employed toluene for the preparation of multi-domain LC films on glass substrates.

Firstly, the polymer was dissolved in toluene, and the solution of low concentration was dropped and spread onto the glass substrate. As toluene was evaporated at room temperature, the polymer in the toluene solution on the glass substrate was transformed from an isotropic solution to a concentrated state with lyotropic LC phase. Complete evaporation of toluene afforded pure polymer thin film with liquid crystal order. This can be referred to as evaporation liquid crystal order formation (ELF). The optical texture of the polymer film is shown in Figure 4(a). The multi-domain polymer has the structure of smectic A $(\mathrm{SmA})$, based on the diamond structure observable in the multi-domain fan-shaped texture. 
Mechanical Orientation in Thermotropic Liquid Crystal State and Magnetic Orientation in Solvent Evaporation Process via Lyotropic Liquid Crystal State of an Amphotropic Low-Bandgap Liquid Crystalline $\pi$-Conjugated Polymer

Hiromasa Goto, ${ }^{1, *}$ Aohan Wang, ${ }^{1}{ }^{1}$ Shigeki Nimori, ${ }^{2}$ Kohsuke Kawabata Liquid Crystal, 2013, 40(9), 1159-1166.
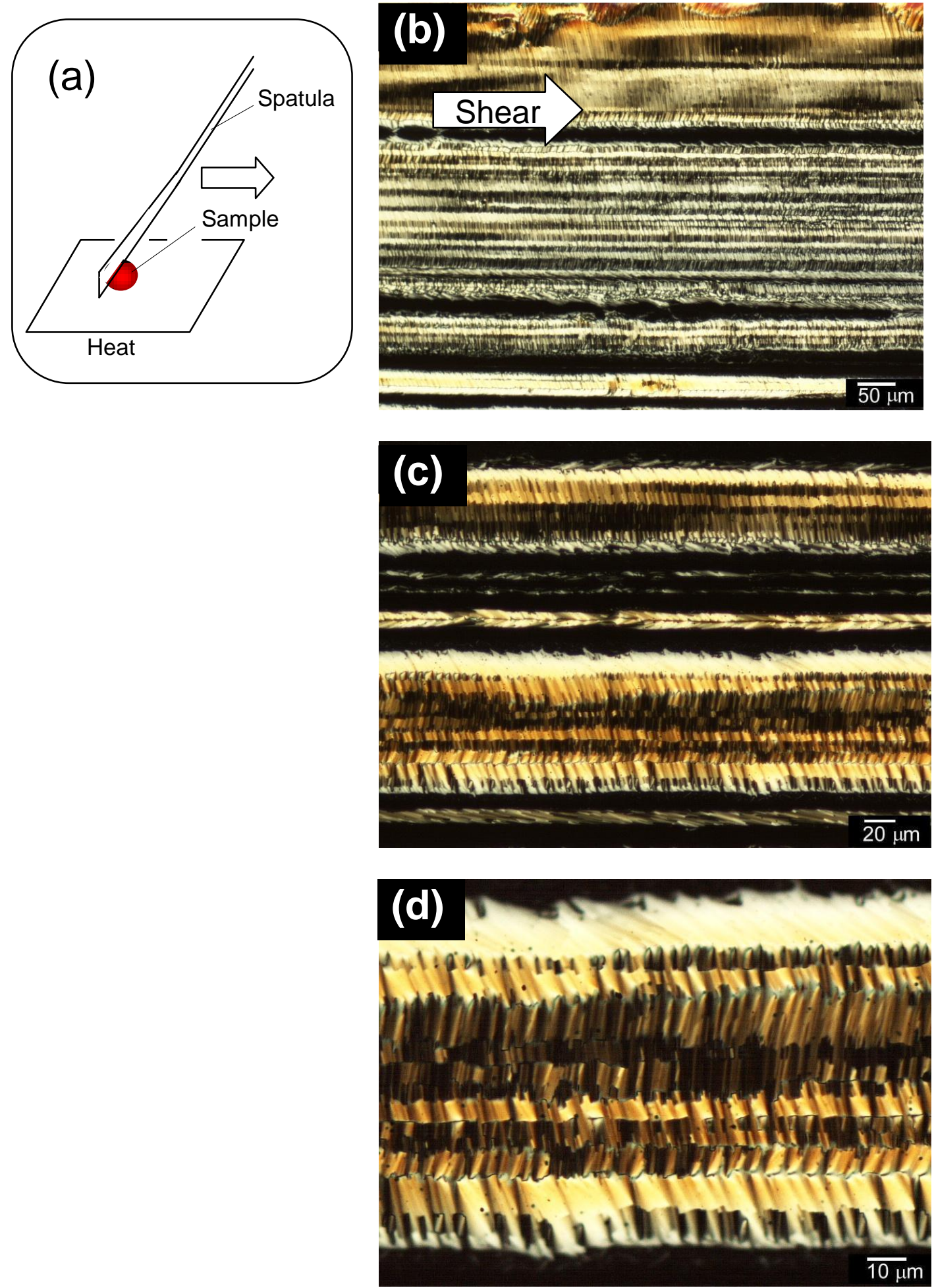

Figure 3. Polarising optical microscopy (POM) image of the oriented polyITN-LC (thin sample) obtained under shear stress in thermotropic liquid-crystalline state. The orientation was accomplished by mechanically moving the metal spatula on thesubstrate. (a) Orientation of the sample by using a spatula to obtain oriented thick sample, (b) 100x, (c) 200x and (d) 500x. 
Mechanical Orientation in Thermotropic Liquid Crystal State and Magnetic Orientation in Solvent Evaporation Process via Lyotropic Liquid Crystal State of an Amphotropic Low-Bandgap Liquid Crystalline $\pi$-Conjugated Polymer

Hiromasa Goto, ${ }^{1, *}$ Aohan Wang, ${ }^{1}{ }^{1}$ Shigeki Nimori, ${ }^{2}$ Kohsuke Kawabata

Liquid Crystal, 2013, 40(9), 1159-1166.

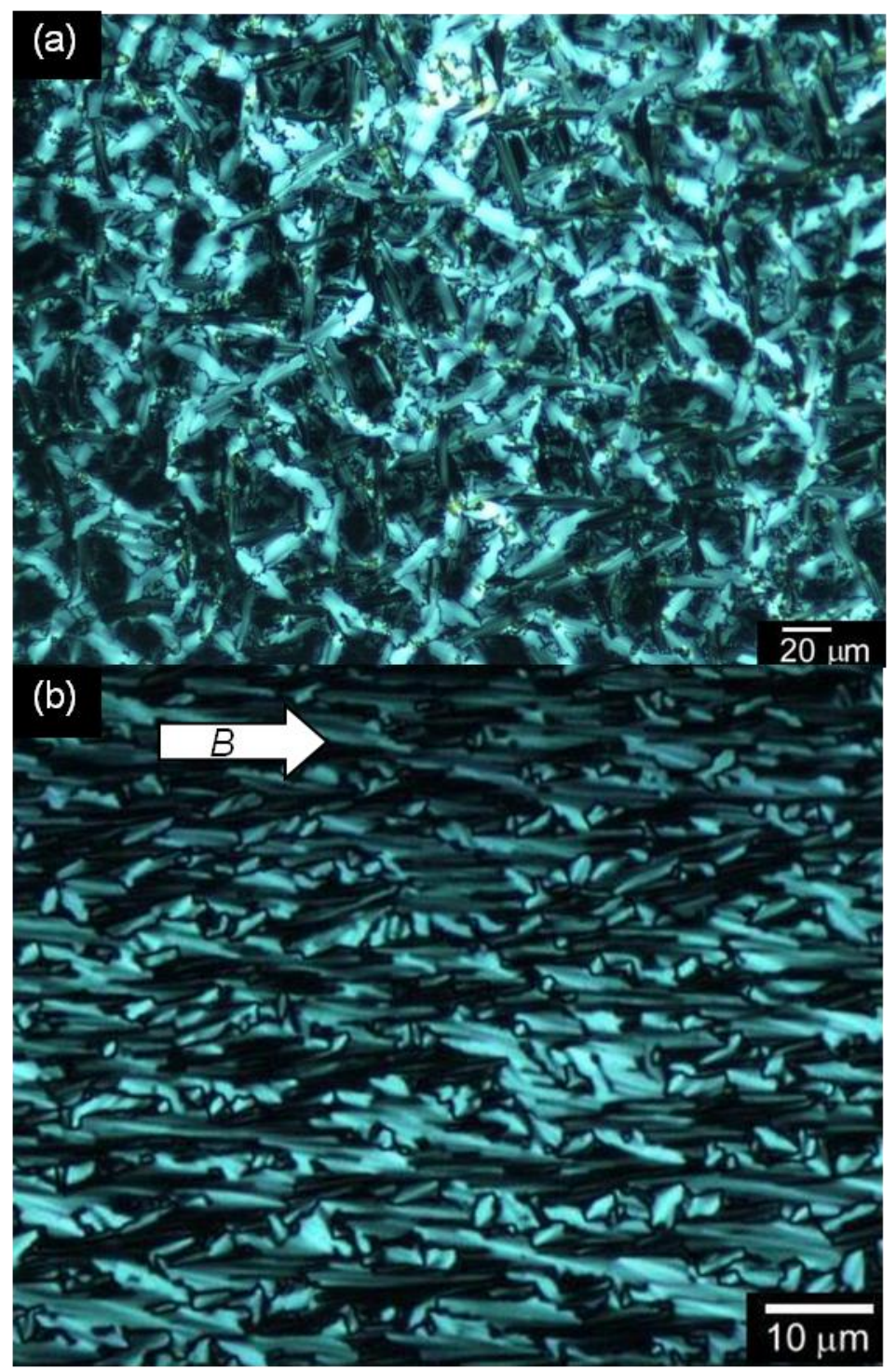

Figure 4. Polarizing optical microscopy (POM) images of polyITN-LC (very thin sample) with lyotropic liquid crystal order. (a) Non-oriented sample obtained in the absence of magnetic field. (b) polyITN-LC with lyotropic LC order oriented in the magnetic field of $12 \mathrm{~T}$.

\section{Orientation of thermotropic LC}

Figure 5 shows polarizing optical absorption spectra of the oriented sample. In the case of the thick oriented sample, absorption intensity of the side chain that appeares at short wavelengths in the vertical direction to the shear stress is strengthened (Figure 5(a)). The anisotropy $\left(\mathrm{Abs}_{\|} / \mathrm{Abs}_{\perp}\right)$ is found to be 1.26 at $420 \mathrm{~nm}$ (the $\pi-\pi^{*}$ transition of the 
Mechanical Orientation in Thermotropic Liquid Crystal State and Magnetic Orientation in Solvent Evaporation Process via Lyotropic Liquid Crystal State of an Amphotropic Low-Bandgap Liquid Crystalline $\pi$-Conjugated Polymer

Hiromasa Goto, ${ }^{1, *}$ Aohan Wang, ${ }^{1}{ }^{1}$ Shigeki Nimori, ${ }^{2}$ Kohsuke Kawabata

Liquid Crystal, 2013, 40(9), 1159-1166.

main chain). The main chain may align perpendicular to the side chains because the polymer shows the SmA structure. The side chain and the main chain absorption features cannot be separated in the absorption spectra at short wavelengths. A possible orientation is illustrated in Figure 6(a).

In the case of the thin oriented sample, the absorption intensity in the vertical direction as compared to the shear stress direction is weakened (Figure 5(b)). The side chains of polyITN-LC of the thin film sample align in the vertical direction against shear stress via formation of the LC band structure, and the main chains align parallel to the shear direction (Figure 6(b)).
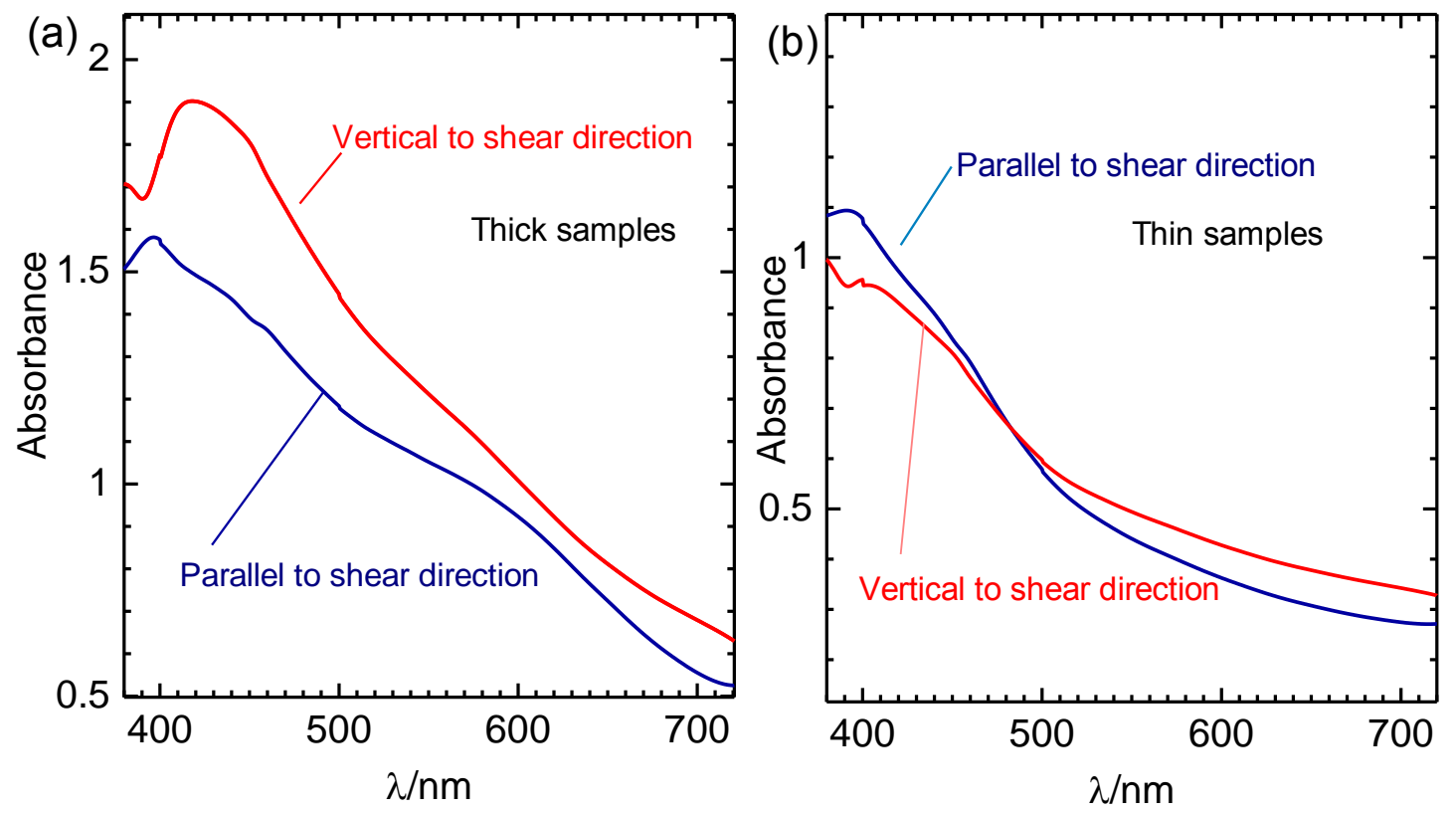

Figure 5. Polarized absorption spectra of polyITN-LC. (a) Thick oriented sample prepared by moving two glass plates (sandwiched cell) to apply shear stress. (b) Thin oriented sample prepared by moving spatula on the substrate to apply shear stress. 
Mechanical Orientation in Thermotropic Liquid Crystal State and Magnetic Orientation in Solvent Evaporation Process via Lyotropic Liquid Crystal State of an Amphotropic Low-Bandgap Liquid Crystalline $\pi$-Conjugated Polymer

Hiromasa Goto, ${ }^{1, *}$ Aohan Wang, ${ }^{1}{ }^{1}$ Shigeki Nimori, ${ }^{2}$ Kohsuke Kawabata Liquid Crystal, 2013, 40(9). 1159-1166.

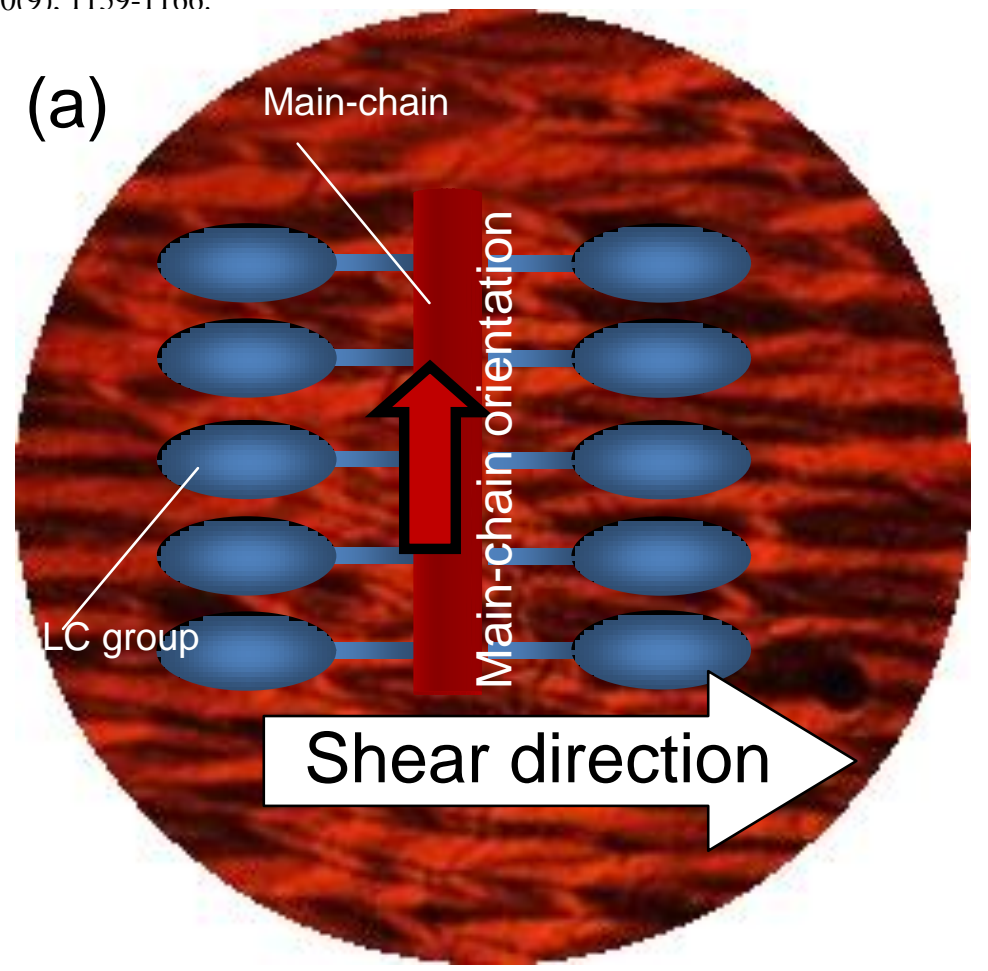

\section{Thick sample}

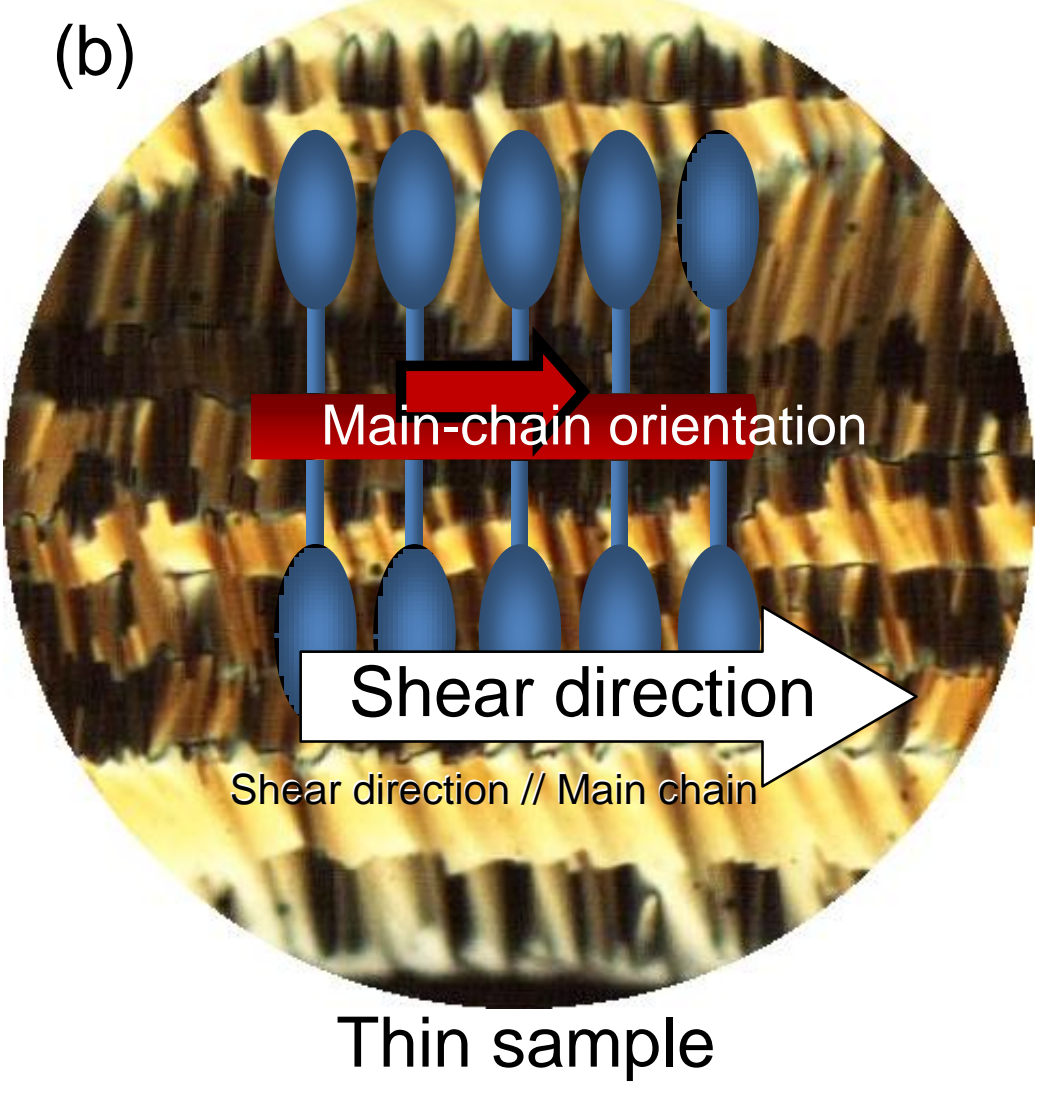

Figure 6. Plausible structure of the oriented sample generated by applying shear stress.

(a) Thick oriented sample prepared by using two glass plates. (b) Thin oriented sample prepared by using spatula to apply shear stress. 
Mechanical Orientation in Thermotropic Liquid Crystal State and Magnetic Orientation in Solvent Evaporation Process via Lyotropic Liquid Crystal State of an Amphotropic Low-Bandgap Liquid Crystalline $\pi$-Conjugated Polymer

Hiromasa Goto, ${ }^{1, *}$ Aohan Wang, ${ }^{1}{ }^{1}$ Shigeki Nimori, ${ }^{2}$ Kohsuke Kawabata

Liquid Crystal, 2013, 40(9), 1159-1166.

\section{Orientation in lyotropic LC under magnetic field}

The present polymer shows lyotropic LC character at certain concentrations. In the present stage, a sufficient sample amount of polyITN-LC to determine the lyotropic LC concentration range cannot be prepared. Firstly, thin polyITN-LC in toluene solution was prepared. The solvent in the polymer solution was gradually evaporated at room temperature. The polymer forms solid films with lyotropic LC order via a certain lyotropic LC concentration range in the evaporation process. The polymer shows no crystalline phase after completion of the evaporation. Bulk samples of polyITN-LC (as-prepared sample) show birefringence, because the bulk state of the polyITN-LC forms liquid crystal ordered structure before the formation of the thin cast film (Figure S2, Supplementary Information).

We also developed a method of magnetic orientation in solvent evaporation process via LC state for the alignment of polyITN-LC. High viscosity of polyITN-LC in the thermotropic LC state resulted in the lack of orientation in the magnetic field of $12 \mathrm{~T}$. On the other hand, uniaxial alignment of polyITN-LC in lyotropic LC state under magnetic field was achieved.

PolyITN-LC was dissolved in a large amount of toluene $(<10 \%)$. The dilute polymer solution shows no lyotropic LC. The solution was applied dropwise to a glass substrate and placed in a magnetic field. The toluene solvent was evaporated in the magnetic field accompanied by progressive molecular alignment. In this case, the growth of the LC domains and magnetic orientation occur simultaneously in this process. After completion of the evaporation from the lyotropic LC, the polymer forms thin solid films showing LC order with uniaxial alignment, as shown in Figure 4(b), where the SmA domains are aligned along the magnetic field. This result indicated that the side-chains of the polyITN-LC are aligned along the magnetic field resulting in the formation of uniaxial orientation. The main chains are aligned in the vertical direction against the magnetic field, accompanied by the orientation of the side chains along the magnetic field. It should be noted that the thickness of the film with LC order can be tuned by the concentration of the polymer solution. The relatively thick sample can be aligned along the magnetic field of $12 \mathrm{~T}$ with this technique (LME), as shown in Figure 7. The sample shows optical retardation and uniaxial alignment along the magnetic field resulting in a fish-shoal optical texture. The magnetic field aligns the side chains of 
Mechanical Orientation in Thermotropic Liquid Crystal State and Magnetic Orientation in Solvent Evaporation Process via Lyotropic Liquid Crystal State of an Amphotropic Low-Bandgap Liquid Crystalline $\pi$-Conjugated Polymer

Hiromasa Goto, ${ }^{1, *}$ Aohan Wang, ${ }^{1}{ }^{1}$ Shigeki Nimori, ${ }^{2}$ Kohsuke Kawabata

Liquid Crystal, 2013, 40(9), 1159-1166.

polyITN-LC in the LC state, and the main chains are aligned perpendicularly to the magnetic field.

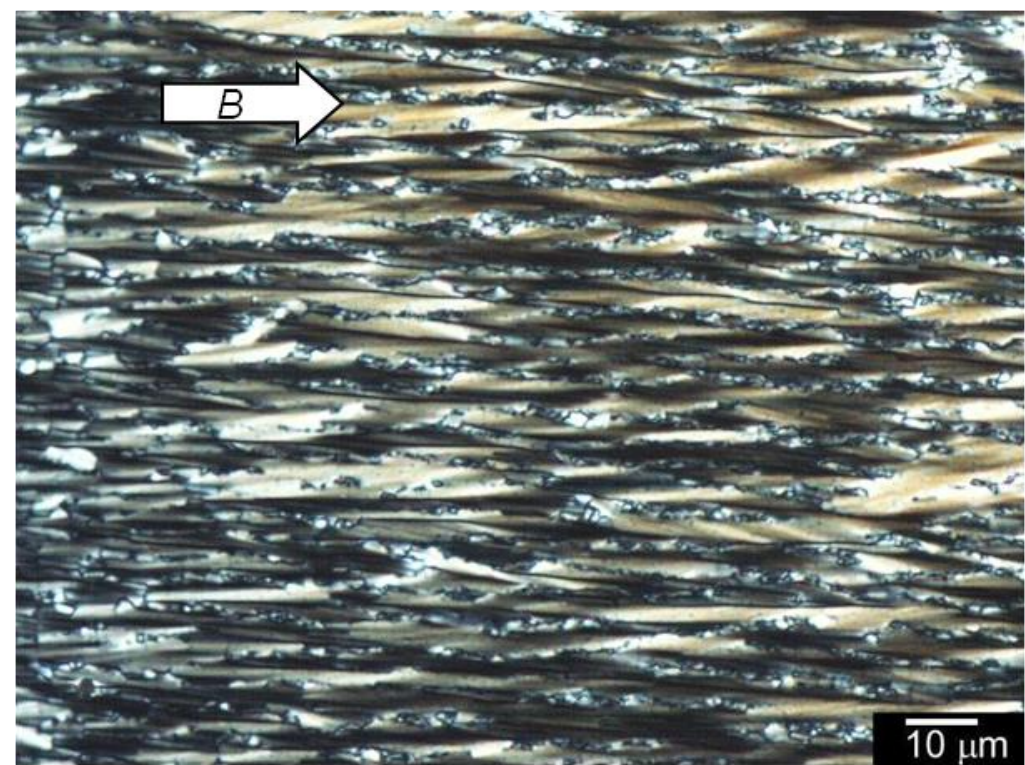

Figure 7. Polarizing optical microscopy (POM) image of the oriented polyITN-LC

(thick sample) with lyotropic liquid crystal order showing fish-shoal texture.

\section{Conclusions}

Molecular orientation by applying shear stress and magnetic field were achieved. The thin sample formed by spatula scratching on the glass substrate produced an LC band structure with a chevron pattern. The orientations of the thick sample and the thin sample under application of shear stress were different. The magnetic orientation in evaporation method developed in this research allows production of uniaxially oriented polymer films exhibiting aligned domain structure.

\section{Acknowledgments}

We would like to thank Chemical Analysis Division, Research Facility Center for Science and Technology, University of Tsukuba, and the Glass Workshop of University of Tsukuba. Pyrimidine-type LC precursors were generously provided by Midori Chemical (Midori Kagaku, Japan). We wish to express our sincere appreciation to Dr. R.H.L. Kiebooms for encouragement and advice. 
Mechanical Orientation in Thermotropic Liquid Crystal State and Magnetic Orientation in Solvent Evaporation Process via Lyotropic Liquid Crystal State of an Amphotropic Low-Bandgap Liquid Crystalline $\pi$-Conjugated Polymer

Hiromasa Goto, ${ }^{1, *}$ Aohan Wang, ${ }^{1}{ }^{1}$ Shigeki Nimori, ${ }^{2}$ Kohsuke Kawabata

Liquid Crystal, 2013, 40(9), 1159-1166.

\section{References}

(1) Tseng H.B.; Ying L.; Hsu B.B.Y.; Perez L.A.; Takacs C.J.; Bazan G.C.; Heeger A.J. High mobility field effect transistors based on macroscopically oriented regioregular copolymers. Nano Letters 2012;12:6353-6357.

(2) Gu X, Liu ZJ, Yao JK.; Boe Technology Group Co Ltd. A liquid crystal panel and manufacturing method thereof LCD (Liquid crystal display) panel and manufacturing method thereof. China patent CN102650759 A, 201229 Aug.

(3) Geary J.M.; Goodby J.W.; Kmetz A.R.; Patel J.S. The mechanism of polymer alignment of liquid - crystal materials. J. Appl. Phys. 1987;62:4100-4108.

(4) Schadt M, Chigrinov VG, KoK S, Nose K, Nagashima Y, Nishiyama I, Takatsu H.; DIC Corporation. Optical anisotropic body. Jpn patent JP2012058511, 2012 May 22.

(5) Kang S.H.; Na J.H.; Moon S.N.; Lee W.I.; Yoo P.J.; Lee S.D. Self-organized anisotropic wrinkling of molecularly aligned liquid crystalline polymer. Langmuir $2012 ; 28: 3576-3582$.

(6) Lee J.J.; Kim E.J.; Paek S.H. Improvement of LC alignment by control of UV irradiation and wet cleaning for a photo-degradable polyimide layer. Mol. Crys. Liq. Crys. 2011;539:196-201.

(7) Samitsu S.; Takanishi Y.; Yamamoto J. Self Assembly and one-dimensional alignment of a conducting polymer nanofiber in a nematic liquid Crystal. Macromolecules 2009;42:4366-4368.

(8) Bradley D.D.C. Conjugated polymer electroluminescence. Synth. Met. $1993 ; 54: 401-415$.

(9) Wu X.; Liu Y.Q.; Zhu D.B. Synthesis and characterizaiton of a new conjugated polymer containing cyano substituents for light-emitting diodes. J. Mat. Chem 2011;11:1327-1331.

(10) Greenham N.C.; Friend R.H.; Bradley D.D.C. Angular dependence of the emission from a conjugated polymer light-emitting diode: Implications for efficiency calculations. Adv. Mater. 1994;6:491-494.

(11) Sun M. L.; Jiang X.; Liu W.; Zhu T.B.; Huang F.; Cao Y. Selenophene and fluorene based narrow band gap copolymers with $\mathrm{Eg}=1.41 \mathrm{eV}$ for near infrared polymer light emitting diodes.Synth. Met. 2012;162:1406-1410. 
Mechanical Orientation in Thermotropic Liquid Crystal State and Magnetic Orientation in Solvent Evaporation Process via Lyotropic Liquid Crystal State of an Amphotropic Low-Bandgap Liquid Crystalline $\pi$-Conjugated Polymer

Hiromasa Goto, ${ }^{1, *}$ Aohan Wang, ${ }^{1}{ }^{1}$ Shigeki Nimori, ${ }^{2}$ Kohsuke Kawabata

Liquid Crystal, 2013, 40(9), 1159-1166.

(12) Chen L.; Shen X.X.; Chen Y.W. A novel thiophene derivative-based conjugated polymer for polymer solar cells with high open-circuit voltage. Chin. J. Chem. 2012;30:2219-2224.

(13) Zhang Z.G.; Zhang S.Y.; Min J.; Cui C.H.; Geng H.; Shuai Z.G.; Li Y.F. Side chain engineering of polythiophene derivatives with a thienylene-vinylene conjugated side chain for application in polymer solar cells. Macromolecules 2012;45:2312-2320.

(14) Cardona C.M.; Li W.; Kaifer A.E.; Stockdale D.; Bazan G.C. Electrochemical considerations for determining absolute frontier orbital energy levels of conjugated polymers for solar cell applications. Adv. Mater. 2011;23:23672371.

(15) Morel M.; Aguilera C.; Jiménez V.; Basáez L.; Gebhardt P.; Heggie, S. Synthesis and properties of new liquid crystals derivatives of 2,7-diethynyl-9,9-dihexylfluorene. Liq. Cryst. 2012;39:847-856.

(16) Billa, M.T.; Kassireddy K.; Haro M.; Al-Kalifah M.S.; Kelly S.M.; Kitney S.P.; O'Neill, M. Liquid crystalline organic semiconductors: nematic spiro[cyclopentyl-1,9']fluorenes. Liq. Cryst. 2011;38:813-829.

(17) Kim, J.; Park J.; Jung Y.-J.;, Kim E.-C.; Ahn T.; Ka J.-W, Yi M.H. Synthesis and characterisation of photopolymerisable liquid crystals based on the $\pi$-extended fluorene core and their corresponding non-reactive analogues. Liq. Cryst. 2011;38:589-599.

(18) Goto H.; Nimori S. Liquid crystal electropolymerisation under magnetic field and resultant linear polarised electrochromism. J. Mater. Chem., 2010;20:1891-1898.

(19) Kawabata K.; Yoneyama H.; Goto H. Uniaxially ordered conjugated polymer film prepared by electrochemical polymerization in a nematic liquid crystal with rubbing orientation method showing redox-driven tunable dichroism. Polym. Chem., 2010;1:1606-1608.

(20) Higashi T.; Yamasaki N.; Utsumi H.; Yoshida H.; Fujii A.; Ozaki M. Fabrication of aligned liquid-crystalline $\pi$-conjugated polymer films by controlling solution-casting patters. Int. Conf. Sci. Tech. Synth. Met., Atlanta, USA (July $8-13,2012)$. 
Mechanical Orientation in Thermotropic Liquid Crystal State and Magnetic Orientation in Solvent Evaporation Process via Lyotropic Liquid Crystal State of an Amphotropic Low-Bandgap Liquid Crystalline $\pi$-Conjugated Polymer

Hiromasa Goto, ${ }^{1, *}$ Aohan Wang, ${ }^{1}{ }^{1}$ Shigeki Nimori, ${ }^{2}$ Kohsuke Kawabata

Liquid Crystal, 2013, 40(9), 1159-1166.

(21) Dacke M.; Nordstrom P.; Scholtz C.H.; Warrant E.J. A specialized dorsal rim area for polarized light detection in the compound eye of the scarab beetle Pachysoma striatum. J. Comp. Physiol. A 2002;188:211-216.

(22) Warrant E.J. Polarisation vision: beetles see circularly polarised light. Current Biology 2012;20:610-612.

(23) (a) Chiou T.H.; Place A.R.; Caldwell R.L.; Marshall N.J.; Cronin T.W.; A novle function for a carotenoid: astaxanthin used as a polarizer for visual signalling in a mantis shrimp. J. Exp. Bio 2012;215:584-589. (b) Chiou T.H.; Kleinlogel S.; Cronin T.; Caldwell R.; Loeffler B.; Siddiqi A.; Goldizen A.; Marshall J. Circular polarisation vision in a stomatopod crustacean. Curr. Biol. 2008;18:429-434.

(24) Brink D.J.; van der Berg N.G.; Prinsloo L.C. The role of interface effects on the reflection of circularly polarised light from the thin-film structure of scarabus beetles. Surf. Int. Anal. 2008;40:769-771.

(25) Goto, H.; Wang, A.; Kawabata, K. Synthesis and Properties of Low-Bandgap Liquid Crystalline $\pi$-Conjugated Polymers, J. Mater. Sci., 2013; 48: 7523-7532. 
Mechanical Orientation in Thermotropic Liquid Crystal State and Magnetic Orientation in Solvent Evaporation Process via Lyotropic Liquid Crystal State of an Amphotropic Low-Bandgap Liquid Crystalline $\pi$-Conjugated Polymer

Hiromasa Goto, ${ }^{1, *}$ Aohan Wang, ${ }^{1}{ }^{1}$ Shigeki Nimori, ${ }^{2}$ Kohsuke Kawabata Liquid Crystal, 2013, 40(9), 1159-1166.

Supplementary Information for:

Mechanical Orientation in Thermotropic Liquid Crystal State and Magnetic Orientation in Solvent Evaporation Process via Lyotropic Liquid Crystal State of an Amphotropic Low-Bandgap Liquid Crystalline $\pi$-Conjugated Polymer

Hiromasa Goto, ${ }^{1, *}$ Aohan Wang, ${ }^{1}$ Kohsuke Kawabata, ${ }^{1}$ Shigeki Nimori ${ }^{2}$

${ }^{1}$ Division of Materials Science, Faculty of Pure and Applied Sciences, University of Tsukuba, Tsukuba, Ibaraki 305-8573, Japan

${ }^{2}$ Tsukuba Magnet Laboratory, National Institute for Materials Science, Tsukuba, Ibaraki, 305-0003, Japan

*Correspondence to H. Goto, email: gotoh@ims.tsukuba.ac.jp, fax: +81-298-53-4490 
Mechanical Orientation in Thermotropic Liquid Crystal State and Magnetic Orientation in Solvent Evaporation Process via Lyotropic Liquid Crystal State of an Amphotropic Low-Bandgap Liquid Crystalline $\pi$-Conjugated Polymer

Hiromasa Goto, ${ }^{1, *}$ Aohan Wang, ${ }^{1}{ }^{1}$ Shigeki Nimori, ${ }^{2}$ Kohsuke Kawabata Liquid Crystal, 2013, 40(9), 1159-1166.

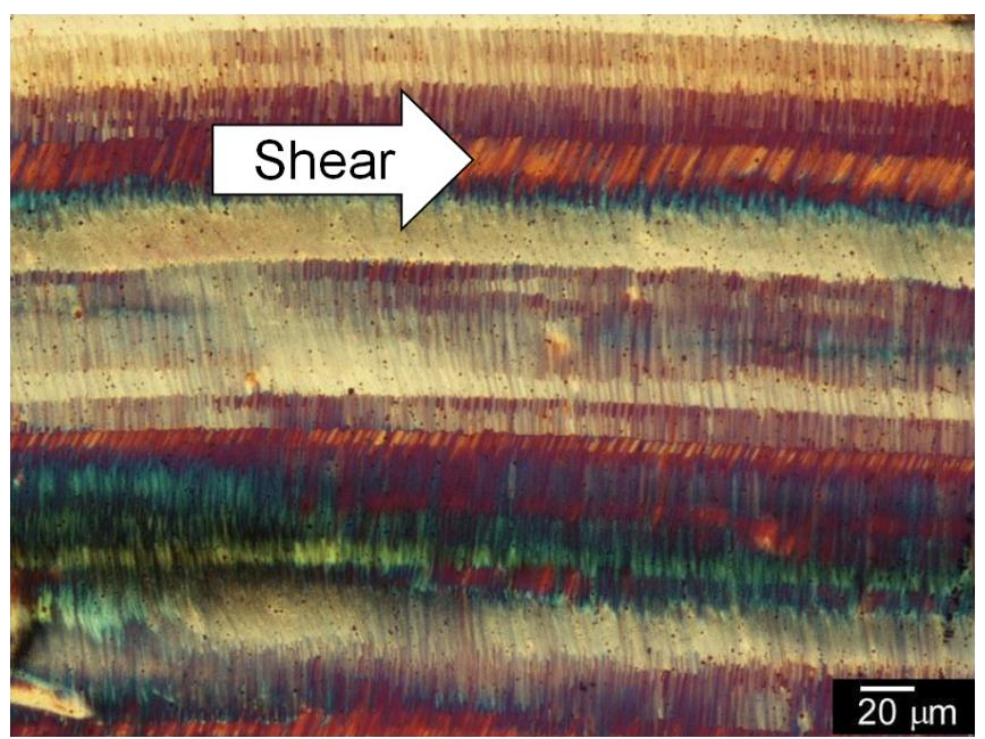

Figure S1. Polarizing optical microscopy (POM) image of the oriented polyITN-LC (thin sample) with shear stress with insertion of gypsum first-order red plate. 
Mechanical Orientation in Thermotropic Liquid Crystal State and Magnetic Orientation in Solvent Evaporation Process via Lyotropic Liquid Crystal State of an Amphotropic Low-Bandgap Liquid Crystalline $\pi$-Conjugated Polymer

Hiromasa Goto, ${ }^{1, *}$ Aohan Wang, ${ }^{1}{ }^{1}$ Shigeki Nimori, ${ }^{2}$ Kohsuke Kawabata

Liquid Crystal, 2013, 40(9), 1159-1166.

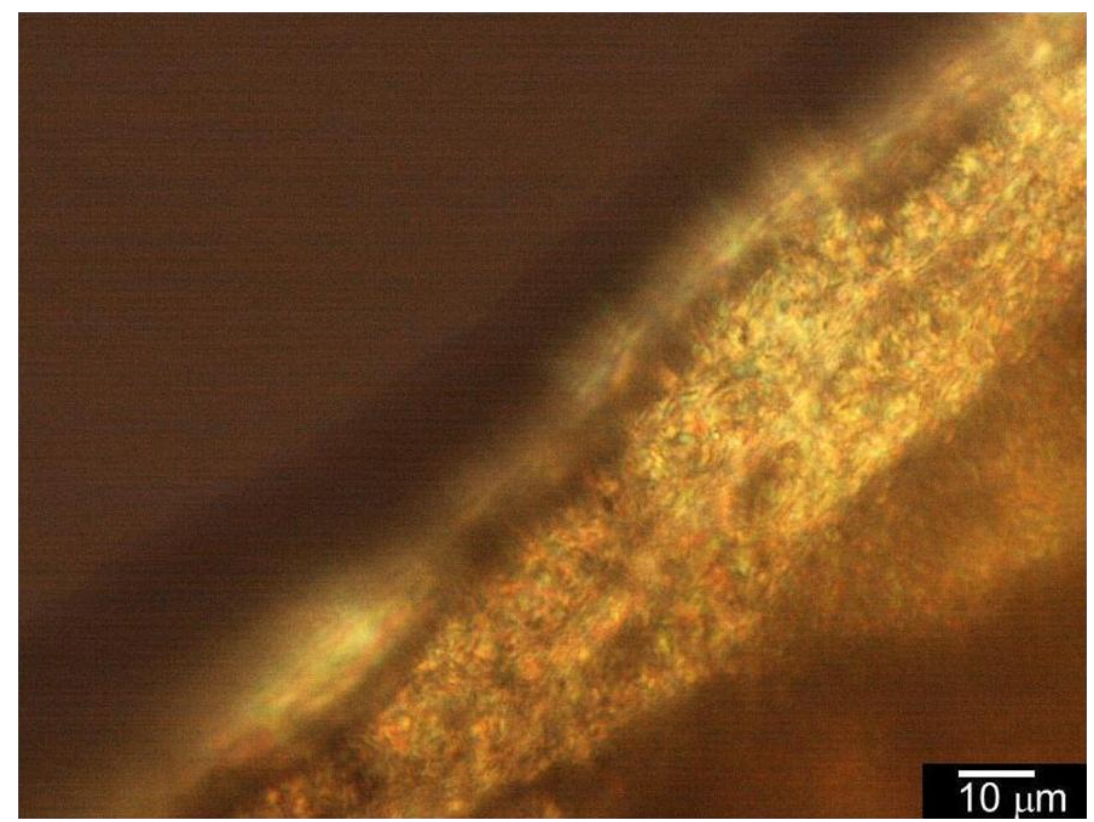

Figure S2. Polarizing optical microscopy (POM) image of the bulk sample of polyITN-LC after purification in synthesis. 